TRANSACTIONS OF THE

AMERICAN MATHEMATICAL SOCIETY

Volume 359, Number 2, February 2007, Pages 897-908

S 0002-9947(06)03921-3

Article electronically published on September 11, 2006

\title{
SYZYGIES USING VECTOR BUNDLES
}

\author{
MONTSERRAT TEIXIDOR I BIGAS
}

\begin{abstract}
This paper studies syzygies of curves that have been embedded in projective space by line bundles of large degree. The proofs take advantage of the relationship between syzygies and spaces of section of vector bundles associated to the given line bundles.
\end{abstract}

\section{INTRODUCTION}

Let $C$ be a projective irreducible non-singular curve of genus $g$ defined over a field of characteristic zero. Let $L$ be a line bundle of degree at least $2 g+1$ on $C$ and consider the immersion of $C$ in projective space associated to the complete linear series $|L|$. Define the rings

$$
R=\bigoplus_{n=0}^{\infty} H^{0}(\mathbf{P}, \mathcal{O}(n)), S=\bigoplus_{n=0}^{\infty} H^{0}\left(C, L^{n}\right)
$$

Then, $R$ is a graded $S$ module and admits a minimal graded free resolution.

$$
0 \rightarrow F_{t} \rightarrow \ldots \rightarrow F_{1} \rightarrow F_{0} \rightarrow 0 .
$$

The vector space of syzygies $K_{p, q}$ is defined as the piece of degree $q$ of $F_{p}$.

In the case of a curve, one easily sees (cf. for example [Ei] or Proposition 3.1) that $K_{p, q}=0$ if $q \geq 3$ or $q=0, p \neq 0$. Hence, one is left with only two strands corresponding to $q=1,2$. Moreover, these two strands are related (cf. for example Proposition 3.2), so in fact it suffices to compute one of them.

For curves of high degree, one expects that $K_{p, 1}=0$ if $p$ is large. On the other hand, if the curve has special linear series $L_{1}, L_{2}$ of dimensions $r_{1}, r_{2}$ and $L=$ $L_{1} \otimes L_{2}$, then from the converse of Green's conjecture (see GL1]), $K_{r_{1}+r_{2}-1,1} \neq 0$.

The question then arises as to whether the non-vanishing of the $K_{p, 1}$ can be explained by the existence of certain linear series on the curve. A conjecture of Green and Lazarsfeld (see GL2, Conjecture 3.7) states that this is the case: Let $L$ be a line bundle of sufficiently high degree giving rise to a linear series of dimension $r$; then $K_{r-d, 1}(L)=0$ unless $C$ is $d$-gonal.

This conjecture has been proved recently for the generic curve ( $\mathrm{AV}, \mathrm{A}]$ ) or equivalently, for the curves of largest possible gonality. In this paper we deal with the other end of the spectrum, namely the curves with small gonality. In Proposition 3.7. we provide a new proof of Green's $K_{p, 1}$ Theorem (see [G], Theorem 3.c.1, p. $151)$ in the case of curves. In 3.8, we reprove Ehbauer's Theorem (Eh, Theorem 1.4 , p. 146). While the results contained here are not new, we do provide new proofs and a simpler, unified presentation.

Received by the editors March 24, 2004 and, in revised form, January 7, 2005.

2000 Mathematics Subject Classification. Primary 14H51, 14H15, 14M60.

(C)2006 American Mathematical Society 897

Reverts to public domain 28 years from publication 


\section{Preliminaries}

It is standard that the $K_{p, q}$ can be computed by taking homology in the middle term of the Koszul complex

$$
\begin{aligned}
\wedge^{p+1} H^{0}(L) \otimes H^{0}\left(C, L^{q-1}\right) & \rightarrow \wedge^{p} H^{0}(L) \otimes \\
& H^{0}\left(C, L^{q}\right) \\
& \rightarrow \wedge^{p-1} H^{0}(L) \otimes H^{0}\left(C, L^{q+1}\right) \rightarrow \ldots
\end{aligned}
$$

Now define a vector bundle $E_{L}$ by using the exact sequence

$$
0 \rightarrow E_{L}^{*} \rightarrow H^{0}(L) \otimes \mathcal{O}_{C} \rightarrow L \rightarrow 0 .
$$

In order to compare this with the Koszul complex, we consider the wedge powers of the sequence above

$$
0 \rightarrow \wedge^{p} E_{L}^{*} \otimes L^{q} \rightarrow \wedge^{p} H^{0}(L) \otimes L^{q} \rightarrow \wedge^{p-1} E_{L}^{*} \otimes L^{q+1} \rightarrow 0
$$

and the analogous sequences obtained by replacing $p, q$ by $p+1, q-1$ and $p-1, q+1$. The map in the Koszul sequence

$$
\ldots \wedge^{p+1} H^{0}(L) \otimes H^{0}\left(L^{q-1}\right) \rightarrow \wedge^{p} H^{0}(L) \otimes H^{0}\left(L^{q}\right) \ldots
$$

can be factored through $H^{0}\left(\wedge^{p} E^{*} \otimes L^{q}\right)$. The two factoring maps

$$
\wedge^{p+1} H^{0}(L) \otimes H^{0}\left(L^{q-1}\right) \rightarrow H^{0}\left(\wedge^{p} E_{L}^{*} \otimes L^{q}\right)
$$

and

$$
H^{0}\left(\wedge^{p} E_{L}^{*} \otimes L^{q}\right) \rightarrow \wedge^{p} H^{0}(L) \otimes H^{0}\left(L^{q}\right)
$$

are obtained by taking homology on the sequences above. One then obtains the following presentations for the syzygies.

\subsection{Lemma.}

$K_{p, q}=\frac{H^{0}\left(\wedge^{p} E_{L}^{*} \otimes L^{q}\right)}{I m\left[\wedge^{p+1} H^{0}(L) \otimes H^{0}\left(L^{q-1}\right)\right]}=\operatorname{Im}\left[H^{0}\left(\wedge^{p} E_{L}^{*} \otimes L^{q}\right) \rightarrow H^{1}\left(\wedge^{p+1} E_{L}^{*} \otimes L^{q-1}\right)\right]$.

Notation. In all that follows, we will assume that $d \geq 2 g+1$ and that $L$ is a line bundle of degree $d$ on $C$. We define $r$ by $h^{0}(L)=d+1-g=r+1$. We have a natural immersion $C \rightarrow \mathbf{P}^{r}$. We will use freely the identifications given in Lemma 2.1 for various values of $p, q$.

We shall write $E$ instead of $E_{L}$ when $L$ is clear from the context.

The first statement in the lemma below was proved by David Butler in $[\mathrm{B}$, Theorem 1.2. The second follows from the first because in characteristic zero the wedge powers of a stable bundle are semistable.

2.2. Lemma. Under the above conditions on degree, the vector bundle $E_{L}$ is stable. Hence, in characteristic zero, its wedge powers are semistable.

\section{Syzygies of CURVES OF LARGE DEgREE}

3.1. Proposition. $K_{p, 0}=0, p \neq 0, \operatorname{dim} K_{0,0}=1, K_{p . q}=0$ if $q \geq 3$.

Proof. The exact sequence $(*)$ corresponding to $p+1,0$ becomes

$$
0 \rightarrow \wedge^{p+1} E^{*} \otimes L^{-1} \rightarrow \wedge^{p+1} H^{0}(L) \otimes L^{-1} \rightarrow \wedge^{p} E^{*} \rightarrow 0 .
$$

If $p>0$, then $h^{0}\left(\wedge^{p} E^{*}\right)=0$, as this is a semistable sheaf of negative degree. Hence, from the first description of $K_{p, 0}$ in Lemma 2.1, $K_{p, 0}=0$.

If $p=0, H^{0}\left(\wedge^{p} E^{*}\right)=H^{0}(\mathcal{O})$ has dimension one while $H^{0}(L) \otimes H^{0}\left(L^{-1}\right)=0$. Hence, $\operatorname{dim} K_{0,0}=1$. 
Assume now $p \neq 0, q \geq 3$. As $\operatorname{rank} E=d-g=r$, if $p+1>r, \wedge^{p+1} E=0$. If $p+1 \leq r$, the slope

$$
\mu\left(\wedge^{p+1} E^{*} \otimes L^{q-1}\right)=(q-1) d-\frac{(p+1) d}{r}=d\left(q-1-\frac{p+1}{r}\right) \geq d>2 g-2 .
$$

Hence, $h^{1}\left(\wedge^{p+1} E^{*} \otimes L^{q-1}\right)=0$. Then, from the second description of $K_{p, q}$ in Lemma 2.1, the result follows.

3.2. Proposition. $\operatorname{dim} K_{p .2}-\operatorname{dim} K_{p+1,1}$ depends only on $d$ and not on $L$ (and can be explicitly calculated).

Proof. Taking $q=1$ and $p+1$ instead of $p$ in the exact sequence $(*)$, we get

$$
0 \rightarrow \wedge^{p+2} E^{*} \rightarrow \wedge^{p+2} H^{0}(L) \otimes \mathcal{O} \rightarrow \wedge^{p+1} E^{*} \otimes L \rightarrow 0 .
$$

As $\wedge^{p+2} E^{*}$ is semistable of negative degree, its space of sections is zero. Hence, from the first description of $K_{p+1,1}$ in Lemma 2.1

$$
\operatorname{dim} K_{p+1,1}=h^{0}\left(\wedge^{p+1} E^{*} \otimes L\right)-\left(\begin{array}{c}
r+1 \\
p+2
\end{array}\right) .
$$

On the other hand, from

$$
\begin{gathered}
0 \rightarrow \wedge^{p+1} E_{L}^{*} \otimes L \rightarrow \wedge^{p+1} H^{0}(L) \otimes L \rightarrow \wedge^{p} E^{*} \otimes L^{2} \rightarrow 0 \\
\operatorname{dim} K_{p, 2}=h^{0}\left(\wedge^{p} E^{*} \otimes L^{2}\right)-(r+1)\left(\begin{array}{l}
r+1 \\
p+1
\end{array}\right)+h^{0}\left(\wedge^{p+1} E^{*} \otimes L\right) .
\end{gathered}
$$

Therefore,

$$
\operatorname{dim} K_{p, 2}-\operatorname{dim} K_{p+1,1}=h^{0}\left(\wedge^{p} E^{*} \otimes L^{2}\right)+\left(\begin{array}{c}
r+1 \\
p+2
\end{array}\right)-(r+1)\left(\begin{array}{c}
r+1 \\
p+1
\end{array}\right) .
$$

As $\wedge^{p} E^{*} \otimes L^{2}$ has slope greater than $2 g-2$, the dimension of its space of sections is independent of $L$ for a fixed $d$, namely $h^{0}\left(\wedge^{p} E^{*} \otimes L^{2}\right)=\left(\begin{array}{l}r \\ p\end{array}\right)\left(2 d-\frac{p d}{r}+1-g\right)$. Then

$$
K_{p, 2}-K_{p+1,1}=\left(\begin{array}{l}
r \\
p
\end{array}\right)\left(2 d-\frac{p d}{r}+1-g\right)+\left(\begin{array}{c}
r+1 \\
p+2
\end{array}\right)-(r+1)\left(\begin{array}{c}
r+1 \\
p+1
\end{array}\right) .
$$

\subsection{Proposition. $\operatorname{dim} K_{r-1,2}=g$.}

Proof (see also Ei], Proposition 8.6). As $h^{0}(L)=r+1, \wedge^{r+1} H^{0}(L)$ is isomorphic to the base field and $\wedge^{r} H^{0}(L)$ is naturally isomorphic to $\left(H^{0}(L)\right)^{*}$. As $r k E=r$ and $\wedge^{r} E=L$, then $\wedge^{r-1} E^{*}=E \otimes L^{-1}$. Using these isomorphisms, the exact sequence

$$
0 \rightarrow \wedge^{r} E^{*} \otimes L \rightarrow \wedge^{r} H^{0}(L) \otimes L \rightarrow \wedge^{r-1} E^{*} \otimes L^{2} \rightarrow 0
$$

can be written as

$$
0 \rightarrow \mathcal{O} \rightarrow H^{0}(L)^{*} \otimes L \rightarrow E \otimes L \rightarrow 0 .
$$

Taking homology, one obtains

$$
H^{0}(L)^{*} \otimes H^{0}(L) \rightarrow H^{0}(E \otimes L) \rightarrow H^{1}(\mathcal{O}) \rightarrow 0 .
$$

Hence, $\operatorname{dim} K_{r-1,2}=h^{1}(\mathcal{O})=g$

Remark. From the second interpretation in Lemma 2.1, $K_{p, q}=0$ if $p \geq r$. Then, the result also follows from Proposition 3.2 . 
3.4. Proposition. For $C$ a non-rational curve, $K_{r-1,1}=0$.

Proof. Again, we use the isomorphisms

$$
\wedge^{k} E^{*} \cong \wedge^{r-k} E \otimes L^{-1}, \wedge^{k} H^{0}(L)=\wedge^{r+1-k} H^{0}(L)^{*} .
$$

The exact sequence

$$
0 \rightarrow \wedge^{r} E^{*} \rightarrow \wedge^{r} H^{0}(L) \otimes \mathcal{O} \rightarrow \wedge^{r-1} E^{*} \otimes L \rightarrow 0
$$

becomes

$$
0 \rightarrow L^{*} \rightarrow H^{0}(L)^{*} \otimes \mathcal{O} \rightarrow E \rightarrow 0 .
$$

Taking homology, this gives

$$
0 \rightarrow H^{0}(L)^{*} \rightarrow H^{0}(E) \rightarrow H^{1}\left(L^{*}\right) .
$$

Hence, the statement is equivalent to the surjectivity of the map $H^{0}(L)^{*} \rightarrow H^{0}(E)$. By the injectivity of this map, it suffices to prove that $h^{0}(E)=r+1$. This fact is proved as follows (see GL3, diagram 2.1): Let $P_{1}, \ldots, P_{r-1}$ be generic points, $D=P_{1}+\ldots+P_{r-1}$. Then, $h^{0}(L(-D))=2$. Consider the diagram

$$
\begin{aligned}
& 0 \rightarrow L^{-1}(D) \rightarrow H^{0}(L(-D)) \otimes \mathcal{O} \rightarrow L(-D) \rightarrow 0 \\
& 0 \rightarrow \begin{array}{c}
\downarrow \\
E^{*}
\end{array} \quad \rightarrow \quad H^{0}(L) \otimes \mathcal{O} \quad \rightarrow \quad \stackrel{\downarrow}{L} \quad \rightarrow \quad 0 \\
& 0 \rightarrow \quad \bar{E}^{*} \quad \rightarrow \quad \frac{H^{0}(L)}{H^{0}(L(-D))} \otimes \mathcal{O} \quad \rightarrow \quad L_{D} \quad \rightarrow \quad 0
\end{aligned}
$$

Here $\bar{E}^{*}$ is defined as the cokernel of the left vertical sequence. It can then be shown that it is also the kernel of the bottom row. From this description, it follows that $\bar{E}^{*}=\bigoplus_{i=1}^{r-1} \mathcal{O}\left(-P_{i}\right)$. We then get an exact sequence

$$
0 \rightarrow \bigoplus_{i=1}^{r-1} \mathcal{O}\left(P_{i}\right) \rightarrow E \rightarrow L(-D) \rightarrow 0
$$

Therefore,

$$
h^{0}(E) \leq \sum h^{0}\left(\mathcal{O}\left(P_{i}\right)\right)+h^{0}(L(-D))=r-1+2=r+1,
$$

as required.

3.5. Proposition. Let $C$ be a curve immersed by a line bundle $L$ of degree $2 g+1+k$. Then, if $p \leq k, K_{p, 2}=0$.

Proof (see $\left[\mathrm{L}\right.$, section 1). From the second interpretation of $K_{p, 2}$ in Lemma 2.1, it suffices to show that $h^{1}\left(\wedge^{p+1} E^{*} \otimes L\right)=0$.

From the proof of Proposition 3.4, we have an exact sequence

$$
0 \rightarrow L^{-1}(D) \rightarrow E^{*} \rightarrow \bigoplus_{i=1}^{r-1} \mathcal{O}\left(-P_{i}\right) \rightarrow 0,
$$

where $D=\bigoplus_{i=1}^{r-1} P_{i}$ is a divisor made of generic points. Taking wedge powers, we obtain exact sequences

$$
0 \rightarrow \bigoplus L^{-1}\left(D-P_{i_{1}}-\ldots-P_{i_{p}}\right) \rightarrow \wedge^{p+1} E^{*} \rightarrow \bigoplus \mathcal{O}\left(-P_{i_{1}}-\ldots-P_{i_{p+1}}\right) \rightarrow 0 .
$$

Tensoring with $L$, this gives rise to

$$
0 \rightarrow \bigoplus \mathcal{O}\left(D-P_{i_{1}}-\ldots-P_{i_{p}}\right) \rightarrow \wedge^{p+1} E^{*} \otimes L \rightarrow \bigoplus L\left(-P_{i_{1}}-\ldots-P_{i_{p+1}}\right) \rightarrow 0 .
$$


Given a choice of points $P_{i_{1}} \ldots P_{i_{p+1}}$ denote by $P_{j_{1}} \ldots P_{j_{r-1-p}}$ the complementary set of points in $D$. Then, $h^{1}\left(D\left(-P_{i_{1}}-\ldots-P_{i_{p+1}}\right)\right)=h^{1}\left(P_{j_{1}}+\ldots+P_{j_{r-1-p}}\right)=0$ if and only if (by the genericity of the points) $r-1-p \geq g$. This condition can be written as $p \leq r-1-g=d-2 g-1=k$ and is satisfied by assumption. Moreover, $\operatorname{deg} K \otimes L^{-1}\left(P_{i_{1}}+\ldots+P_{i_{p+1}}\right)=p-k-2<0$. Then,

$$
0=h^{0}\left(K \otimes L^{-1}\left(P_{i_{1}}+\ldots+P_{i_{p+1}}\right)\right)=h^{1}\left(L\left(-P_{i_{1}}-\ldots-P_{i_{p+1}}\right)\right) .
$$

Hence, from the exact sequence above, $h^{1}\left(\wedge^{p+1} E^{*} \otimes L\right)=0$ if $p \leq k$.

We have seen in Proposition 3.4 that $K_{r-1,1}=0$. We want to see that $K_{p, 1}=0$ if $p$ is large enough and the curve is sufficiently general. The following lemma will be useful.

3.6. Lemma. $K_{p, 1}=0$ if and only if $h^{0}\left(\wedge^{r-p} E\right)=\left(\begin{array}{c}r+1 \\ r-p\end{array}\right)$.

Proof. From Lemma 2.1,$K_{p, 1}=0$ if and only if the natural map $\wedge^{p+1} H^{0}(L) \rightarrow$ $H^{0}\left(\wedge^{p} E^{*} \otimes L\right)$ is surjective. The kernel of this map is $H^{0}\left(\wedge^{p+1} E^{*}\right)$, and this space of sections is zero because $\wedge^{p+1} E^{*}$ is a semistable vector bundle of negative degree. Therefore, $K_{p, 1}=0$ if and only if $h^{0}\left(\wedge^{p} E^{*} \otimes L\right)=\left(\begin{array}{c}r+1 \\ p+1\end{array}\right)$.

As rankE $=r$ and $\wedge^{r} E=L$, one has an isomorphism of $\wedge^{p} E^{*} \otimes L$ with $\wedge^{r-p} E$. Moreover, $\left(\begin{array}{c}r+1 \\ p+1\end{array}\right)=\left(\begin{array}{l}r+1 \\ r-p\end{array}\right)$, and the result follows. V.

The proof of the next two propositions is inspired from the paper of Claire Voisin

3.7. Proposition. Let $L$ be a line bundle of degree at least $2 g+1$ on a curve of genus at least four. Then $K_{r-2,1}=0$ unless $C$ is trigonal and $L=K\left(g_{3}^{1}\right)$ or $C$ is hyperelliptic.

Proof. We shall see at the end that the assumptions on $L$ imply that there exists a divisor $D=P_{1}+\ldots+P_{r}$ satisfying the following conditions:

a) $h^{0}(L(-D))=h^{0}\left(L\left(-\left(D-P_{i}\right)\right)=2\right.$ for every $i=1, \ldots, r$.

b) $h^{0}\left(P_{i_{1}}+P_{i_{2}}+P_{i_{3}}\right)=1$ for all triples of points in the support of $D$.

We now show that these conditions imply that $h^{0}\left(\wedge^{2}(E)\right)=\left(\begin{array}{c}r+1 \\ 2\end{array}\right)$ and therefore $K_{r-2: 1}=0$ by Lemma 3.6 .

Consider the following exact diagram:

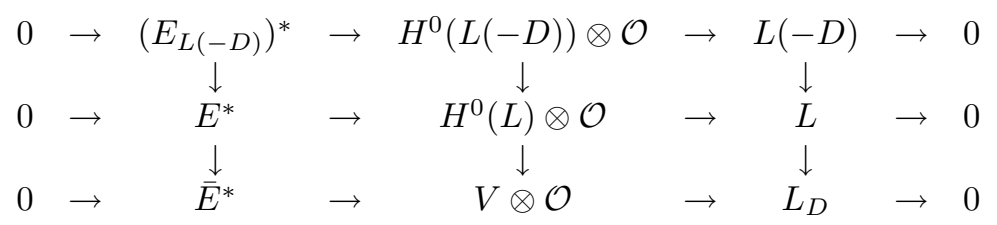

where $V=\frac{H^{0}(L)}{H^{0}(L(-D))}$.

As $h^{0}(L(-D))=2,\left(E_{L(-D)}\right)^{*}=L^{-1}(D)$. The condition $h^{0}\left(L\left(-\left(D-P_{i}\right)\right)\right)=$ $h^{0}(L)-\operatorname{deg}\left(D-P_{i}\right)$ implies that $h^{0}\left(L\left(-\left(D-P_{i}-P_{j}\right)\right)\right)=h^{0}\left(L\left(-\left(D-P_{i}\right)\right)\right)+1=3$. Take a section $s$ of $H^{0}\left(L\left(-\left(D-P_{1}-P_{2}\right)\right)\right)-H^{0}\left(L\left(-\left(D-P_{i}\right)\right)\right)$. The image of $s$ 
in the quotient $V$ is non-zero and $\operatorname{dim} \frac{V}{\langle s\rangle}=r-2$. We then have

$$
\begin{aligned}
& 0 \rightarrow \mathcal{O}\left(-P_{1}-P_{2}\right) \rightarrow\langle s\rangle \otimes \mathcal{O} \quad \rightarrow \quad L_{P_{1}+P_{2}} \quad \rightarrow 0
\end{aligned}
$$

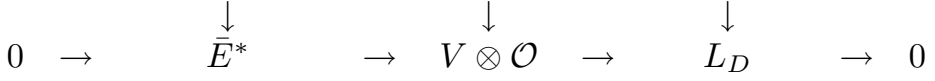

$$
\begin{aligned}
& 0 \rightarrow \quad A \quad \rightarrow \quad \frac{V}{\langle s\rangle} \otimes \mathcal{O} \rightarrow L_{P_{3}+\ldots+P_{r}} \rightarrow 0
\end{aligned}
$$

From the diagram, $A=\mathcal{O}\left(-P_{3}\right) \oplus \ldots \oplus \mathcal{O}\left(-P_{r}\right)$. We then get exact sequences

$$
\begin{gathered}
0 \rightarrow \bigoplus_{i=3}^{r} \mathcal{O}\left(P_{i}\right) \rightarrow \bar{E} \rightarrow \mathcal{O}\left(P_{1}+P_{2}\right) \rightarrow 0, \\
0 \rightarrow \bar{E} \rightarrow E \rightarrow L(-D) \rightarrow 0 .
\end{gathered}
$$

Taking the second wedge power of the latter, we obtain

$$
0 \rightarrow \wedge^{2} \bar{E} \rightarrow \wedge^{2} E \rightarrow \bar{E} \otimes L(-D) \rightarrow 0 .
$$

From the first exact sequence, we get

$$
\begin{aligned}
0 & \rightarrow \bigoplus_{i=3}^{r} L\left(-\left(D-P_{i}\right)\right) \rightarrow \bar{E} \otimes L(-D) \rightarrow L\left(-D+P_{1}+P_{2}\right) \rightarrow 0 \\
0 & \rightarrow \bigoplus_{3 \leq i_{1}<i_{2} \leq r} \mathcal{O}\left(P_{i_{1}}+P_{i_{2}}\right) \rightarrow \wedge^{2} \bar{E} \rightarrow \bigoplus_{i=3}^{r} \mathcal{O}\left(P_{1}+P_{2}+P_{i}\right) \rightarrow 0 .
\end{aligned}
$$

Hence,

$$
\begin{gathered}
h^{0}\left(\wedge^{2} E\right) \leq h^{0}\left(\wedge^{2} \bar{E}\right)+h^{0}(\bar{E} \otimes L(-D)) \leq \sum_{3 \leq i_{1}<i_{2} \leq r} h^{0}\left(\mathcal{O}\left(P_{i_{1}}+P_{i_{2}}\right)\right) \\
+\sum_{i=3}^{r} h^{0}\left(\mathcal{O}\left(P_{1}+P_{2}+P_{i}\right)\right)+\sum_{i=3}^{r} h^{0}\left(L\left(-\left(D-P_{i}\right)\right)\right)+h^{0}\left(L\left(-D+P_{1}+P_{2}\right)\right) \\
=\left(\begin{array}{c}
r-2 \\
2
\end{array}\right)+r-2+2(r-2)+3 \\
=\left(\begin{array}{c}
r-2 \\
2
\end{array}\right)\left(\begin{array}{l}
3 \\
0
\end{array}\right)+\left(\begin{array}{c}
r-2 \\
1
\end{array}\right)\left(\begin{array}{l}
3 \\
1
\end{array}\right)+\left(\begin{array}{c}
r-2 \\
0
\end{array}\right)\left(\begin{array}{l}
3 \\
2
\end{array}\right)=\left(\begin{array}{c}
r+1 \\
2
\end{array}\right) .
\end{gathered}
$$

It remains to show that a divisor $D$ exists satisfying conditions a) and b) above. Let $D_{g-2}$ be a generic effective divisor of degree $g-2$. Let $D$ be an effective divisor of the complete linear series $\left|L \otimes K^{-1}\left(D_{g-2}\right)\right|$. This exists as $\operatorname{deg}\left(L \otimes K^{-1}\left(D_{g-2}\right)\right)=$ $d-g \geq g+1$, and therefore $L \otimes K^{-1}\left(D_{g-2}\right)$ has sections. Moreover, $h^{0}(L(-D))=$ $h^{0}\left(K\left(-D_{g-2}\right)\right)=2$. We now show that for every point $P_{i}$ in the support of $D$, $h^{0}\left(L\left(-\left(D-P_{i}\right)\right)\right)=2$. As $L\left(-\left(D-P_{i}\right)\right)=K\left(-D_{g-2}+P_{i}\right)$, this is equivalent to $h^{0}\left(D_{g-2}-P_{i}\right)=0$. From our choice, $h^{0}\left(D_{g-2}\right)=1$. Hence we are asking that $D, D_{g-2}$ have disjoint supports.

Such a $D$ will exist if $h^{0}\left(L \otimes K^{-1}\left(D_{g-2}\right)\right)>h^{0}\left(L \otimes K^{-1}\left(D_{g-2}-Q\right)\right)$ for each $Q$ in the support of $D_{g-2}$. This condition is equivalent to $h^{0}\left(K^{2} \otimes L^{-1}\left(-D_{g-2}\right)\right)=$ $h^{0}\left(K^{2} \otimes L^{-1}\left(-\left(D_{g-2}-Q\right)\right)\right)$. By the genericity of $D_{g-2}$ this happens precisely when $h^{0}\left(K^{2} \otimes L^{-1}\right) \leq g-3$. Assume on the contrary that $h^{0}\left(K^{2} \otimes L^{-1}\right) \geq g-2$. We shall prove that this leads to one of the situations we excluded for $L$. We obtain

$$
g-2 \leq h^{0}\left(K^{2} \otimes L^{-1}\right)=h^{0}\left(L \otimes K^{-1}\right)+3 g-3-d \leq h^{0}\left(L \otimes K^{-1}\right)+g-4 .
$$


Hence, $h^{0}\left(L \otimes K^{-1}\right) \geq 2$. It follows that $K^{2} \otimes L^{-1}$ contributes to the Clifford index of $C$ as $g \geq 4$ and

$$
\begin{aligned}
0 & \leq \operatorname{Cliff}\left(K^{2} \otimes L^{-1}\right)=4 g-4-d-2\left(h^{0}\left(K^{2} \otimes L^{-1}\right)-1\right) \\
& \leq 4 g-4-2 g-1-2(g-3) .
\end{aligned}
$$

Therefore, the only two possibilities are $\operatorname{deg} L=2 g+1, h^{0}\left(K^{2} \otimes L^{-1}\right)=g-2$ and $\operatorname{deg} L=2 g+2, h^{0}\left(K^{2} \otimes L^{-1}\right)=g-2$. In the first case, $L \otimes K^{-1}$ gives a $g_{3}^{1}$, in the second it gives a $g_{4}^{2}$. As $g_{4}^{2}$ appear only on hyperelliptic curves and $g_{4}^{2}=2 g_{2}^{1}$, both cases have been excluded. This takes care of condition a).

Condition b) is obviously satisfied if $C$ is not trigonal. If $C$ is trigonal, we can choose a $D$ satisfying b) so long as the linear system $|D|$ is not composed with a $g_{3}^{1}$. If we assume the curve non-hyperelliptic, the $g_{3}^{1}$ is unique for $g \geq 5$ (there may be two of them for genus $g=4$ ). If $|D|$ is composed with the $g_{3}^{1}$, then $L \otimes K^{-1}\left(D_{g-2}\right)=a g_{3}^{1}, a \geq \frac{g+2}{3}$. As the divisor $D_{g-2}$ depends on $g-2$ parameters, this is impossible.

3.8. Proposition. Let $L$ be a line bundle of degree $d \geq 2 g+3$ on a curve $C$ that is not trigonal of genus at least seven. Then $K_{r-3,1}=0$.

Proof. We shall check in a moment that the given conditions imply that there is an effective divisor of degree $r-1 D=P_{1}+\ldots+P_{r-1}$ such that

a) $h^{0}(L(-D))=3=h^{0}\left(L\left(-D+P_{i}\right)\right), i=1, \ldots, r-1$.

b) $h^{0}\left(P_{i_{1}}+P_{i_{2}}+P_{i_{3}}+P_{i_{4}}\right)=1$ for all quadruples of points on the support of $D$.

c) The maps $H^{0}(L(-D)) \otimes H^{0}\left(K\left(-D^{\prime}\right)\right) \rightarrow H^{0}\left(K \otimes L\left(-D-D^{\prime}\right)\right)$ are onto for every divisor $D^{\prime}$ of degree at most three contained in the support of $D$.

Using conditions a)-c), we now show that $h^{0}\left(\wedge^{3} E\right)=\left(\begin{array}{c}r+1 \\ 3\end{array}\right)$. From Lemma 3.6, this suffices to prove the result.

Consider the diagram

$$
\begin{aligned}
& 0 \rightarrow\left(E_{L(-D)}\right)^{*} \rightarrow H^{0}(L(-D)) \otimes \mathcal{O} \quad \rightarrow \quad L(-D) \rightarrow 0 \\
& 0 \rightarrow \quad E^{*} \quad \rightarrow \quad H^{0}(L) \otimes \mathcal{O} \quad \rightarrow \quad \begin{array}{lll}
\downarrow & \rightarrow & \rightarrow
\end{array}
\end{aligned}
$$

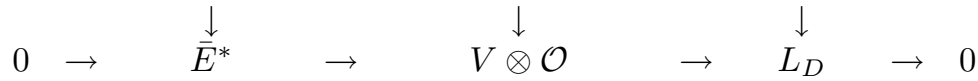

where $V=\frac{H^{0}(L)}{H^{0}(L(-D))}$ is a vector space of dimension $r-2$. We then have the exact sequence

$$
0 \rightarrow \bar{E} \rightarrow E \rightarrow E_{L(-D)} \rightarrow 0,
$$

and $E_{L(-D)}$ is a vector bundle of rank two.

We obtain the bound

$$
h^{0}\left(\wedge^{3} E\right) \leq h^{0}\left(\wedge^{3} \bar{E}\right)+h^{0}\left(\wedge^{2} \bar{E} \otimes E_{L(-D)}\right)+h^{0}\left(\bar{E} \otimes \wedge^{2} E_{L(-D)}\right) .
$$

As $h^{0}(L(-D))=h^{0}(L)-\operatorname{deg} D+1$ and $h^{0}\left(L\left(-\left(D-P_{i}\right)\right)\right)=h^{0}(L(-D))$, it follows that $h^{0}\left(L\left(-\left(D-P_{i}-P_{j}\right)\right)\right)=h^{0}\left(L\left(-\left(D-P_{i}\right)\right)\right)+1=4$. Take a section $s$ of $H^{0}\left(L\left(-\left(D-P_{1}-P_{2}\right)\right)\right)-H^{0}\left(L\left(-\left(D-P_{i}\right)\right)\right)$. The image of $s$ in the quotient $V$ 
is non-zero and $\operatorname{dim} \frac{V}{\langle s\rangle}=r-3$ We have

$$
\begin{aligned}
& 0 \rightarrow \mathcal{O}\left(-P_{1}-P_{2}\right) \quad \rightarrow \quad\langle s\rangle \otimes \mathcal{O} \quad \rightarrow \quad L_{P_{1}+P_{2}} \quad \rightarrow \quad 0
\end{aligned}
$$

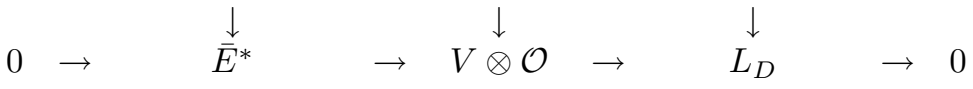

$$
\begin{aligned}
& 0 \rightarrow \bigoplus_{i=3}^{r-1} \stackrel{\downarrow}{\mathcal{O}}\left(-P_{i}\right) \rightarrow \frac{V}{\langle s\rangle} \otimes \mathcal{O} \rightarrow \quad \begin{array}{ccc}
\downarrow & L_{P_{3}+\ldots+P_{r-1}} \rightarrow 0
\end{array}
\end{aligned}
$$

Therefore, the following sequences are exact:

$$
\begin{gathered}
0 \rightarrow \bigoplus_{i=3}^{r-1} \mathcal{O}\left(P_{i}\right) \rightarrow \bar{E} \rightarrow \mathcal{O}\left(P_{1}+P_{2}\right) \rightarrow 0, \\
0 \rightarrow L^{-1}(D) \rightarrow H^{0}(L(-D))^{*} \otimes \mathcal{O} \rightarrow E_{L(-D)} \rightarrow 0 .
\end{gathered}
$$

Taking wedge powers, we get

$$
0 \rightarrow \bigoplus \mathcal{O}\left(P_{i_{1}}+P_{i_{2}}+P_{i_{3}}\right) \rightarrow \wedge^{3} \bar{E} \rightarrow \bigoplus \mathcal{O}\left(P_{i_{1}}+P_{i_{2}}+P_{1}+P_{2}\right) \rightarrow 0 .
$$

Using condition b)

$$
h^{0}\left(\wedge^{3} \bar{E}\right) \leq\left(\begin{array}{c}
r-3 \\
3
\end{array}\right)+\left(\begin{array}{c}
r-3 \\
2
\end{array}\right) .
$$

Also, as $\wedge^{2} E_{L(-D)}=L(-D)$,

$h^{0}\left(\bar{E} \otimes \wedge^{2} E_{L(-D)}\right) \leq \sum_{i=3}^{r-1} h^{0}\left(L\left(-D+P_{i}\right)\right)+h^{0}\left(L\left(-D+P_{1}+P_{2}\right)\right)=3(r-3)+4$.

From

$$
0 \rightarrow \bigoplus \mathcal{O}\left(P_{i_{1}}+P_{i_{2}}\right) \rightarrow \wedge^{2} \bar{E} \rightarrow \bigoplus \mathcal{O}\left(P_{i}+P_{1}+P_{2}\right) \rightarrow 0
$$

one has

$$
h^{0}\left(\wedge^{2} \bar{E} \otimes E_{L(-D)}\right) \leq \sum_{i_{1}, i_{2}} h^{0}\left(E_{L(-D)}\left(P_{i_{1}}+P_{i_{2}}\right)\right)+\sum_{i} h^{0}\left(E_{L(-D)}\left(P_{1}+P_{2}+P_{i}\right)\right) .
$$

In order to compute the second of these numbers, we use the exact sequence

$$
\begin{gathered}
0 \rightarrow L^{-1}\left(D+P_{1}+P_{2}+P_{i}\right) \rightarrow H^{0}(L(-D))^{*} \otimes \mathcal{O}\left(P_{1}+P_{2}+P_{i}\right) \\
\rightarrow E_{L(-D)}\left(P_{1}+P_{2}+P_{i}\right) \rightarrow 0 .
\end{gathered}
$$

The map $H^{1}\left(L^{-1}\left(D+P_{1}+P_{2}+P_{i}\right)\right) \rightarrow H^{0}(L(-D))^{*} \otimes H^{1}\left(\mathcal{O}\left(P_{1}+P_{2}+P_{i}\right)\right)$ is dual of the map

$$
H^{0}\left(K \otimes L\left(-D-P_{1}-P_{2}-P_{i}\right)\right) \leftarrow H^{0}(L(-D)) \otimes H^{0}\left(K\left(-P_{1}-P_{2}-P_{i}\right)\right)
$$

which is surjective by assumption c). As $h^{0}\left(L^{-1}\left(D+P_{1}+P_{2}+P_{i}\right)\right)=0$, we obtain

$$
h^{0}\left(E_{L(-D)}\left(P_{1}+P_{2}+P_{i}\right)\right) \leq h^{0}(L(-D)) h^{0}\left(\mathcal{O}\left(P_{1}+P_{2}+P_{i}\right)\right)=3 .
$$

Similarly

$$
h^{0}\left(E_{L(-D)} \otimes \mathcal{O}\left(P_{i_{1}}+P_{i_{2}}\right)\right) \leq h^{0}(L(-D)) h^{0}\left(\mathcal{O}\left(P_{i_{1}}+P_{i_{2}}\right)\right)=3 .
$$

Hence,

$$
h^{0}\left(\wedge^{2} \bar{E} \otimes E_{L(-D)}\right) \leq 3\left(\begin{array}{c}
r-3 \\
2
\end{array}\right)+3(r-3) .
$$


We then deduce that

$$
\begin{aligned}
& h^{0}\left(\wedge^{3} E_{L}\right) \leq\left(\begin{array}{c}
r-3 \\
3
\end{array}\right)+\left(\begin{array}{c}
r-3 \\
2
\end{array}\right)+4+3(r-3)+3\left(\begin{array}{c}
r-3 \\
2
\end{array}\right)+3(r-3) \\
= & \left(\begin{array}{c}
4 \\
0
\end{array}\right)\left(\begin{array}{c}
r-3 \\
3
\end{array}\right)+\left(\begin{array}{l}
4 \\
1
\end{array}\right)\left(\begin{array}{c}
r-3 \\
2
\end{array}\right)+\left(\begin{array}{l}
4 \\
2
\end{array}\right)\left(\begin{array}{c}
r-3 \\
1
\end{array}\right)+\left(\begin{array}{l}
4 \\
3
\end{array}\right)\left(\begin{array}{c}
r-3 \\
0
\end{array}\right)=\left(\begin{array}{c}
r+1 \\
3
\end{array}\right) .
\end{aligned}
$$

It remains to show that a divisor $D$ exists satisfying conditions a), b), c) above. Let $D_{g-3}$ be a generic effective divisor of degree $g-3$. Choose as $D$ a generic effective divisor of the complete linear series $\left|L \otimes K^{-1}\left(D_{g-3}\right)\right|$. This is possible as $\operatorname{deg}\left(L \otimes K^{-1}\left(D_{g-3}\right)\right)=d-g-1 \geq g+2$. Moreover, $h^{0}(L(-D))=h^{0}\left(K\left(-D_{g-3}\right)\right)=$ 3. We now show that we can choose $D$ so that for every point $P_{i}$ in the support of $D, h^{0}\left(L\left(-\left(D-P_{i}\right)\right)\right)=3$. As $L\left(-\left(D-P_{i}\right)\right)=K\left(-D_{g-3}+P_{i}\right)$, this is equivalent to $h^{0}\left(D_{g-3}-P_{i}\right)=0$. From the genericity of $D_{g-3}, h^{0}\left(D_{g-3}\right)=1$. Hence we are asking that $D, D_{g-3}$ have disjoint supports.

Such a $D$ will exist if $h^{0}\left(L \otimes K^{-1}\left(D_{g-3}\right)\right)>h^{0}\left(L \otimes K^{-1}\left(D_{g-3}-Q\right)\right)$ for each $Q$ in the support of $D_{g-3}$. By Serre duality, this translates into $h^{0}\left(K^{2} \otimes L^{-1}\left(-D_{g-3}\right)\right)=$ $h^{0}\left(K^{2} \otimes L^{-1}\left(-\left(D_{g-3}-Q\right)\right)\right)$. By the genericity of $D_{g-3}$, this is equivalent to $h^{0}\left(K^{2} \otimes L^{-1}\right) \leq g-4$. Assume the opposite, namely $h^{0}\left(K^{2} \otimes L^{-1}\right) \geq g-3$. Then,

$$
h^{0}\left(L \otimes K^{-1}\right) \geq d-2 g+2+1-g+g-3=d-2 g \geq 2 .
$$

It follows that $K^{2} \otimes L^{-1}$ contributes to the Clifford index of $C$ and

$$
0 \leq \operatorname{Cliff}\left(K^{2} \otimes L^{-1}\right)=4 g-4-d-2\left(h^{0}\left(K^{2} \otimes L^{-1}\right)-1\right) \leq 2 .
$$

Hence,

$$
d \leq 4 g-4-2\left(h^{0}\left(K^{2} \otimes L^{-1}\right)-1\right) \leq 2 g+4
$$

Then,

$$
h^{0}\left(L \otimes K^{-1}\right)=d+3-3 g+h^{0}\left(K^{2} \otimes L^{-1}\right) \geq d-2 g .
$$

As we are assuming $\operatorname{deg} L \geq 2 g+3$, the only possibilities are then $\operatorname{deg} L=2 g+$ $3, h^{0}\left(L \otimes K^{-1}\right) \geq 3$ and $\operatorname{deg} L=2 g+4, h^{0}\left(L \otimes K^{-1}\right) \geq 4$.

Therefore, $L=K\left(g_{5}^{2}\right)$, which contradicts the bound on the genus of $C$ or $L=$ $K\left(g_{6}^{3}\right)$, and then the curve is hyperelliptic which contradicts the assumption. This takes care of condition a).

Condition b) is obviously satisfied if $C$ is not 4-gonal. Note that $\operatorname{dim} W_{4}^{1} \leq 1$ for $C$ non-hyperelliptic and equality holds only for $C$ trigonal or bielliptic. If $C$ is 4 -gonal, we can choose a $D$ satisfying b) so long as $L \otimes K^{-1}\left(D_{g-3}\right)$ is not composed with a $g_{4}^{1}$. Otherwise, for $C$ non-trigonal or bielliptic, this would imply that $L \otimes K^{-1}\left(D_{g-3}\right)=k g_{4}^{1}$. This is incompatible with the fact that $L \otimes K^{-1}\left(D_{g-3}\right)$ moves in a $(g-3)$-dimensional family of line bundles. For $C$ bielliptic, $W_{4}^{1}$ is the pullback of the linear series of degree two. Hence, if the $D_{i}$ are divisors of various $g_{4}^{1}, h^{0}\left(D_{1}+\ldots+D_{k}\right) \geq 2 k>k+1$ if $k>1$. This contradicts the statement that $L \otimes K^{-1}\left(D_{g-3}\right)$ is composed with an involution.

Before proving c), we show that $|D|$ has no fixed points and is not composed with an involution. Assume that $|D|$ had fixed points for generic $D_{g-3}$. Then for every divisor $D_{g-3}$ there exists a $P_{D_{g-3}}$ such that

$$
h^{0}\left(L \otimes K^{-1}\left(D_{g-3}\right)\right)=h^{0}\left(L \otimes K^{-1}\left(D_{g-3}-P_{D_{g-3}}\right)\right) .
$$

Equivalently

$$
h^{0}\left(K^{2} \otimes L^{-1}\left(-D_{g-3}\right)\right)=h^{0}\left(K^{2} \otimes L^{-1}\left(P_{D_{g-3}}-D_{g-3}\right)\right)-1 .
$$


This implies in particular that $K^{2} \otimes L^{-1}\left(P_{D_{g-3}}-D_{g-3}\right)$ is effective. As this is a family of dimension at least $g-4$ of line bundles, if they are effective they must have degree at least $g-4$. Hence,

$$
4 g-4-d+1-g+3 \geq g-4 \text {. }
$$

Therefore $d \leq 2 g+4$.

Let us show that the condition $h^{0}\left(K^{2} \otimes L^{-1}\left(P_{D_{g-3}}-D_{g-3}\right)\right) \geq 1$ implies $h^{0}\left(K^{2} \otimes L^{-1}\right) \geq g-3$ : If $P_{D_{g-3}}=P$ is fixed independent of $D_{g-3}$, then $h^{0}\left(K^{2} \otimes L^{-1}(P)\right) \geq g-2$. Hence, $h^{0}\left(K^{2} \otimes L^{-1}\right) \geq g-3$. If $P_{D_{g-3}}$ is generic, then

$$
h^{0}\left(K^{2} \otimes L^{-1}\left(P_{D_{g-3}}-D_{g-3}\right)\right)=h^{0}\left(K^{2} \otimes L^{-1}\left(-D_{g-3}\right)\right) \geq 1 .
$$

Moreover, for a fixed $P, D_{g-3}$ moves in a variety of dimension at least $g-4$. Hence, $h^{0}\left(K^{2} \otimes L^{-1}\right) \geq g-3$.

If $d=2 g+4$, from $h^{0}\left(K^{2} \otimes L^{-1}(P)\right) \geq g-3$ one obtains $h^{0}\left(L \otimes K^{-1}\right) \geq 4$. Then, $L=K\left(g_{6}^{3}\right)$, contradicting the assumption. If $d=2 g+3, h^{0}\left(L \otimes K^{-1}\right) \geq 3$ and $L=K\left(g_{5}^{2}\right)$. In either case, this contradicts the assumptions on $L$.

Note that if we were only assuming that $D_{g-3}$ moves in a space of dimension $g-4$, we would obtain that $D$ has at most one fixed point.

Assume now that $|D|$ were composed with an involution. A fixed curve can have only a finite number of non-rational involutions. As there is a $g-3$ dimensional family of divisors $L \otimes K^{-1}\left(D_{g-3}\right)=\mathcal{O}(D)$, the involution must be rational. So, we have

$$
C \rightarrow \mathbf{P}^{1} \rightarrow \mathbf{P}^{1}
$$

where the first map has degree $a$, the second degree $b$, the composition is the map corresponding to the linear system $|D|$ and $\operatorname{dim}|D|=b$. As $\operatorname{dim} W_{a}^{1} \leq a-2$ and $D_{g-3}$ moves in a variety of dimension $g-3$, we would get $a-2 \geq g-3$. Then, $b+1=h^{0}\left(b g_{a}^{1}\right) \geq a b+1-g$. Hence, $b \leq \frac{g}{a-1} \leq \frac{g}{g-2}<2$. Hence, $b=1$ and the map is not composed with an involution.

This result would still hold under the assumption that $D_{g-3}$ moves in a space of dimension $g-4$.

We now prove c). We first study the case of a divisor $D_{2}$ of degree two on the support of $D$. Consider the map given by the complete linear series $|L(-D)|=$ $\left|K-D_{g-3}\right|$. If $C$ is not hyperelliptic, as $D_{g-3}$ is generic, this is a generic projection of the canonical curve. Hence, it gives rise to a nodal plane curve. Let $\bar{D}_{2}$ be the divisor corresponding to a node, $\left|K-D_{g-3}-\bar{D}_{2}\right| \subset\left|K-D_{g-3}\right|$ the pencil cut on $C$ by the lines through the node.

Consider the product maps

$$
\begin{array}{cccc}
\mu_{\bar{D}_{2}}: & H^{0}\left(K-D_{g-3}-\bar{D}_{2}\right) \otimes H^{0}\left(K-D_{2}\right) & \rightarrow & H^{0}\left(K^{2}\left(-D_{g-3}-D_{2}-\bar{D}_{2}\right)\right) \\
\mu: & H^{0}\left(K\left(-D_{g-3}\right)\right) \otimes H^{0}\left(K-D_{2}\right) & \rightarrow & H^{0}\left(K^{2}\left(-D_{g-3}-D_{2}\right)\right)
\end{array}
$$

From the basepoint-free pencil trick, $\operatorname{Ker} \mu_{\bar{D}_{2}}=H^{0}\left(D_{g-3}-D_{2}+\bar{D}_{2}\right)$.

From $\operatorname{deg} L \geq 2 g+3$, one obtains $\operatorname{deg} D \geq g+2$. Hence, $|D|$ is a linear series of dimension at least two that gives rise to a birational map. Therefore, the monodromy of the hyperplane section is the full symmetric group.

Hence, every pair of points of a generic divisor in $D$ imposes the same number of conditions on $D_{g-3}+\bar{D}_{2}$. Assume $h^{0}\left(D_{g-3}-D_{2}+\bar{D}_{2}\right) \geq 1$. It would then follow that $h^{0}\left(D_{g-3}+\bar{D}_{2}-D\right) \geq 1$, which is impossible. Therefore $h^{0}\left(D_{g-3}+\bar{D}_{2}-D_{2}\right)=0$ 
and $\operatorname{dim}\left(\operatorname{Im} \mu_{\bar{D}_{2}}\right)=2(g-2)=h^{0}\left(K^{2}\left(-D_{g-3}-D_{2}-\bar{D}_{2}\right)\right)$. We prove below that $\bar{D}_{2}$ imposes two independent conditions on $H^{0}\left(K_{C}-D_{2}\right)$. Hence, it imposes at least two independent conditions on the image of $\mu$. Then, $\operatorname{dim} \operatorname{Im} \mu \geq \operatorname{dim} \operatorname{Im} \mu_{\bar{D}_{2}}+2=$ $h^{0}\left(K^{2}\left(-D_{g-3}-D_{2}\right)\right)$.

It remains to show that $\bar{D}_{2}$ imposes independent conditions on $K-D_{2}$. Assume this were not the case. Then, $\bar{D}_{2}+D_{2} \in g_{4}^{1}$. If $C$ is not trigonal or bielliptic, $\operatorname{dim} W_{4}^{1} \leq 0$. But the generic choice of $D_{2}$ makes this impossible. In the bielliptic case, one of the components of $W_{g-1}^{1}$ is the pullback of the set of linear series of degree two on the elliptic curve together with sets of $g-5$ points. Then $\bar{D}_{2}=i\left(D_{2}^{\prime}\right)$, with $i$ the involution on $C$ associated to the elliptic cover. Then $D_{2}+\bar{D}_{2}=g_{4}^{1}$ would imply $D_{2}=D_{2}^{\prime}$ against the condition that we checked that $D_{g-3}$ and $D$ are disjoint. Note that the condition fails for a trigonal curve. For a generic $D_{2}=P+Q$, one can find a pair of points in say $g_{3}^{1}-Q=R+S=\bar{D}_{2}$ such that $h^{0}(P+Q+R+S)=2$.

We now deal with the case of a divisor $D_{3}$ of degree three contained in $D$. By the openess of the condition, it suffices to prove that the map is surjective for a particular divisor $D_{g-3}$. Choose a generic point $M \in W_{g}^{2}$. Choose a generic effective divisor of $|M|$ and write it as $D_{g-3}+\bar{D}_{3}$. Then, $h^{0}\left(K-D_{g-3}-\bar{D}_{3}\right)=2$. Now consider the commutative diagram

$$
\begin{array}{cccc}
\mu_{\bar{D}_{3}}: & H^{0}\left(K\left(-D_{g-3}-\bar{D}_{3}\right)\right) \otimes H^{0}\left(K-D_{3}\right) & \rightarrow & H^{0}\left(K^{2}\left(-D_{g-3}-D_{3}-\bar{D}_{3}\right)\right) \\
& \downarrow & & \downarrow \\
\mu: & H^{0}\left(K\left(-D_{g-3}\right)\right) \otimes H^{0}\left(K-D_{3}\right) & \rightarrow & H^{0}\left(K^{2}\left(-D_{g-3}-D_{3}\right)\right) .
\end{array}
$$

By the basepoint-free pencil trick, the kernel of the top row is $H^{0}\left(D_{g-3}-D_{3}+\bar{D}_{3}\right)$.

As $\operatorname{dim} W_{g}^{2} \geq g-6, \operatorname{dim} C_{g}^{2} \geq g-4$. Hence, $D_{g-3}$ moves in a space of dimension at least $g-4$. It follows from the remarks above that the linear series $|D|$ gives rise to a birational map and has at most one fixed point.

When the linear series has no fixed points, with the same argument as in the case of a divisor of degree two, $H^{0}\left(D_{g-3}-D_{3}+\bar{D}_{3}\right)$ has dimension zero. Assume now that $|D|$ has a fixed point $P$. If $D_{3} \subset D-P$, we deduce $\operatorname{dimh}^{0}\left(D_{g-3}+\bar{D}_{3}-(D-P)\right) \geq 1$ while if $D_{3}=D_{2}+P$, $\operatorname{dimh}^{0}\left(D_{g-3}+\bar{D}_{3}-D\right) \geq 1$. Both are impossible. Therefore, the image of $\mu_{\bar{D}_{3}}$ has dimension $2(g-3)=2 g-6$. We shall prove below that $\bar{D}_{3}$ imposes independent conditions on $K-D_{3}$. Hence, it imposes independent conditions on the image of the cup-product map. It follows then that $\operatorname{dim} \operatorname{Im} \mu \geq$ $2 g-6+3=h^{0}\left(K^{2}\left(-D_{g-3}-D_{3}-\bar{D}_{3}\right)\right)$, as required.

As $\left|\bar{D}_{3}+D_{g-3}\right|$ is a linear series of dimension two, the monodromy associated to the generic hyperplane section is the whole symmetric group. If $\bar{D}_{3}$ does not impose independent conditions on $K-D_{3}$, then any divisor of degree three in $\left|\bar{D}_{3}+D_{g-3}\right|$ fails to impose independent conditions on $K-D_{3}$. Hence, $h^{0}\left(K-D_{3}-\bar{D}_{3}-D_{g-3}\right)=$ $g-5$, which is impossible.

\section{REFERENCES}

[A] M. Aprodu, Green-Lazarsfeld gonality conjecture for a generic curve of odd genus, Inter. Math. Res. Not. 63, (2004), 3409-3416. MR2098645 (2005k:14012)

[AV] M. Aprodu, C. Voisin, Green-Lazarsfeld conjecture for generic curves of large gonality, Comptes Rendus Ac. Scien. 36, (2003), 335-339. MR1976314(2004a:14033)

[B] D. Butler, Normal generation of vector bundles over a curve, J. Differential Geometry 39, (1994), 1-34. MR.1258911 (94k:14024)

[Eh] S. Ehbauer, Syzygies of points in projective space and applications, in "Proceedings of the International Conference, Ravello 1992", ed. by Orechia and Chiantini, Walter de Gruyter, 145-170. MR 1292482(95h:13012) 
[Ei] D. Eisenbud, The geometry of syzygies, Graduate Texts in Math., vol. 229, Springer, 2005. MR.2103875 (2005h:13021)

[G] M. Green, Koszul cohomology and the geometry of projective varieties, J. Diff. Geom. 19, (1984), 125-168. MR0739785 (85e:14022)

[GL1] M. Green, R. Lazarsfeld, The non-vanishing of certain Koszul cohomology groups, J. Diff. Geom. 19, (1984), 168-171.

[GL2] M. Green, R. Lazarsfeld, On the projective normality of complete linear series on an algebraic curve, Invent. Math 83, (1986), 73-90. MR0813583 (87g:14022)

[GL3] M. Green, R. Lazarsfeld, A simple proof of Petri's Theorem on canonical curves in "Geometry Today, Giornate di Geometria, Roma 1984", Birkhauser 1985, 129-142. MR0895152 (88h:14038)

[L] R. Lazarsfeld, A sampling of vector bundle techniques in the study of linear series, in "Lectures on Riemann Surfaces, Trieste 1987", World Scient. Publ., 500-559. MR1082360 (92f:14006)

[M] R. Miranda, Algebraic Curves and Riemann Surfaces, Grad. Studies in Math. 5, A.M.S. 1991. MR 1326604 (96f:14029)

[V] C. Voisin, Courbes tetragonales et cohomologie de Koszul, J. Reine Angew. Math. 387, (1988), 111-121. MR0946352(89e:14036)

Department of Mathematics, Tufts University, Medford, Massachusetts 02155 